\title{
Evaluation of Sinus/Edge-Corrected Zero-Echo-Time-Based Attenuation Correction in Brain PET/MRI
}

\author{
Jaewon Yang ${ }^{1}$, Florian Wiesinger ${ }^{2}$, Sandeep Kaushik ${ }^{3}$, Dattesh Shanbhag ${ }^{3}$, Thomas A. Hope ${ }^{1}$, Peder E.Z. Larson ${ }^{1}$, \\ and Youngho Seo ${ }^{1}$ \\ ${ }^{I}$ Department of Radiology and Biomedical Imaging, University of California, San Francisco, San Francisco, California; ${ }^{2}$ GE Global \\ Research, Munich, Germany; and ${ }^{3}$ GE Global Research, Bangalore, India
}

In brain PET/MRI, the major challenge of zero-echo-time (ZTE)-based attenuation correction (ZTAC) is the misclassification of air/tissue/ bone mixtures or their boundaries. Our study aimed to evaluate a sinus/edge-corrected (SEC) ZTAC $\left(\mathrm{ZTAC}_{\mathrm{SEC}}\right)$, relative to an uncorrected (UC) ZTAC (ZTAC ${ }_{\cup C}$ ) and a CT atlas-based attenuation correction (ATAC). Methods: Whole-body ${ }^{18} \mathrm{~F}-\mathrm{FDG}$ PET/MRI scans were obtained for 12 patients after PET/CT scans. Only data acquired at a bed station that included the head were used for this study. Using PET data from PET/MRI, we applied ZTAC $_{U C}, Z_{\text {ZTAC }}$ SEC, ATAC, and reference CT-based attenuation correction (CTAC) to PET attenuation correction. For $\mathrm{ZTAC}_{\cup \mathrm{C}}$, the bias-corrected and normalized ZTE was converted to pseudo-CT with air $(-1,000 \mathrm{HU}$ for ZTE $<0.2)$, softtissue (42 HU for ZTE $>0.75)$, and bone $(-2,000 \times[Z T E-1]+42 \mathrm{HU}$ for $0.2 \leq \mathrm{ZTE} \leq 0.75$ ). Afterward, in the pseudo-CT, sinus/edges were automatically estimated as a binary mask through morphologic processing and edge detection. In the binary mask, the overestimated values were rescaled below $42 \mathrm{HU}$ for ZTAC $_{\mathrm{SEC}}$. For ATAC, the atlas deformed to MR in-phase was segmented to air, inner air, soft tissue, and continuous bone. For the quantitative evaluation, PET mean uptake values were measured in twenty $1-\mathrm{mL}$ volumes of interest distributed throughout brain tissues. The PET uptake was compared using a paired $t$ test. An error histogram was used to show the distribution of voxel-based PET uptake differences. Results: Compared with CTAC, ZTAC $_{\text {SEC }}$ achieved the overall PET quantification accuracy $(0.2 \% \pm 2.4 \%, P=0.23)$ similar to CTAC, in comparison with ZTAC $\mathrm{UC}(5.6 \% \pm 3.5 \%, P<0.01)$ and ATAC $(-0.9 \% \pm 5.0 \%$, $P=0.03)$. Specifically, a substantial improvement with ZTAC $_{\mathrm{SEC}}$ $(0.6 \% \pm 2.7 \%, P<0.01)$ was found in the cerebellum, in comparison with ZTAC $_{\mathrm{UC}}(8.1 \% \pm 3.5 \%, P<0.01)$ and ATAC $(-4.1 \% \pm 4.3 \%$, $P<0.01)$. The histogram of voxel-based uptake differences demonstrated that $Z_{\text {TAC }}$ SEC reduced the magnitude and variation of errors substantially, compared with $Z T A C_{\cup C}$ and ATAC. Conclusion: ZTAC $_{\text {SEC }}$ can provide an accurate PET quantification in brain $\mathrm{PET} / \mathrm{MRI}$, comparable to the accuracy achieved by CTAC, particularly in the cerebellum.

Key Words: neurology; PET; PET/MRI; ZTE; attenuation correction; brain

J Nucl Med 2017; 58:1873-1879

DOI: 10.2967/jnumed.116.188268

\footnotetext{
Received Dec. 6, 2016; revision accepted Apr. 12, 2017.

For correspondence or reprints contact: Jaewon Yang, Department of Radiology and Biomedical Imaging, University of California, San Francisco, 185 Berry St., Suite 350, San Francisco, CA 94107.

E-mail: jaewon.yang@ucsf.edu

Published online May 4, 2017

COPYRIGHT (C 2017 by the Society of Nuclear Medicine and Molecular Imaging.
}

$\mathbf{T}$ he development of PET combined with MRI (PET/MRI) devices has evolved in recent years, and time-of-flight (TOF) capability has been added to PET/MRI (1). Previous studies have demonstrated the potential of PET/MRI for neurologic imaging to investigate brain function, tumor, and degenerative diseases (2). However, because there is no direct way to derive photon attenuation coefficients from MRI for PET attenuation correction (AC), there have been substantial concerns about PET quantification accuracy with regards to MR-based attenuation correction (MRAC) (3).

MRAC methods are categorized into segmentation-based, atlasbased, and PET emission-based approaches (4). Segmentationbased methods derive AC maps by classifying Dixon-based MR images into tissue classes (e.g., air, lung, fat, and soft tissue) to which uniform (i.e., single value) linear attenuation coefficients are assigned (5). The Dixon-based segmentation disregards bone anatomy due to low proton density and short-lived signals (6), even though PET signals are substantially attenuated in bone structures (7). Atlas-based methods include bone structures into AC maps using a single CT atlas (8), multiple CT atlases (9), or a pair of MR model and skull mask (10) through registration. Despite no physical relationship between MRI and CT, advanced atlas-based methods have proposed a statistical model to convert MR intensities to CT numbers using machine learning methods (i.e., training pairs of matched MR/CT images) (11). Without depending on MRI or atlas images, PET emission-based methods have also demonstrated the clinical feasibility for simultaneous estimation of attenuation and activity maps using TOF emission data (12).

Zero-echo-time (ZTE) MRI has demonstrated a possibility to overcome the drawbacks of missing bone signals (i.e., limitation of Dixon-based segmentation), capturing rapidly decaying bone signals (13). Similarly, ultrashort-echo-time MRI also can capture bone signals, with greater flexibility in image contrast and field of views (FOVs) but with increased acoustic noise and greater sensitivity to gradient fidelity compared with ZTE (14). Ultrashort-echo time is typically used to derive MRAC based on T2* relaxation differences (15). The disadvantage of such $\mathrm{T} 2 *$-based approaches is that they are sensitive to short-T2* values resulting from off-resonance, particularly in regions of large magnetic susceptibility differences such as the sinuses, and other short-T2* tissues such as tendons, all of which may be misclassified as bone. Meanwhile, ZTE-based MRAC methods have used proton-density differences to contrast bone against soft tissues and air (13) and are more robust to off-resonance effects than T2*-based methods.

A preliminary ZTE-based attenuation correction (ZTAC) method was evaluated for brain PET AC in a TOF PET/MRI 
system (SIGNA PETMR; GE Healthcare) (16). The preliminary ZTAC demonstrated more accurate AC in comparison with the atlas-based attenuation correction (ATAC) protocol in the scanner; but the major challenge of ZTAC was the misclassification of air/ tissue/bone mixtures (e.g., sinus or mastoid) or their edges (e.g., skin or trachea) as bone, resulting in overestimated PET quantification. Inaccurate ATAC in the cerebellum is also a well-known problem because the level of sinuses, in which air, soft tissue, and bone structures are mixed, is a challenge for accurate registration (3). Therefore, it is important to improve the accuracy of ZTAC near the skull base for neurologic PET/MRI studies because the targetto-reference ratio, a widely used quantification technique, uses the cerebellum as a reference for PET quantification (17). Our study aimed to evaluate a sinus/edge-corrected (SEC) ZTE-based attenuation correction $\left(\mathrm{ZTAC}_{\mathrm{SEC}}\right.$ ), relative to an uncorrected (UC) ZTAC $\left(\mathrm{ZTAC}_{\mathrm{UC}}\right)$ and the ATAC.

\section{MATERIALS AND METHODS}

\section{Patient Information}

The patient study was approved by the Institutional Review Board, and all patients signed an informed consent form before the examinations. Twelve patients ( 5 men and 7 women) underwent whole-body ${ }^{18} \mathrm{~F}-\mathrm{FDG}$ $\mathrm{PET} / \mathrm{CT}$ for clinical indications, immediately followed by wholebody PET/MRI. There was no second administration of ${ }^{18} \mathrm{~F}-\mathrm{FDG}$ for PET/MRI. The average patient age was $60.8 \pm 9.5 \mathrm{y}$ (range, 44-74 y), the average weight was $71.9 \pm 13.5 \mathrm{~kg}$ (range, 54.4-97.5 kg), and the average administered dose of ${ }^{18} \mathrm{~F}-\mathrm{FDG}$ was $297.9 \pm 58.9 \mathrm{MBq}$ (range, 218.3-392.2 MBq). For PET/MRI, the average scan duration of the whole brain was $245.0 \pm 92.5 \mathrm{~s}$ (range, 135-390 s), and the average time difference between injection and scan was $147.1 \pm 23.7 \mathrm{~min}$ (range, 119.6-188.6 min). No pathology in the brain was reported. Only PET/MRI/CT data acquired at a bed station that includes the head were used for this study.

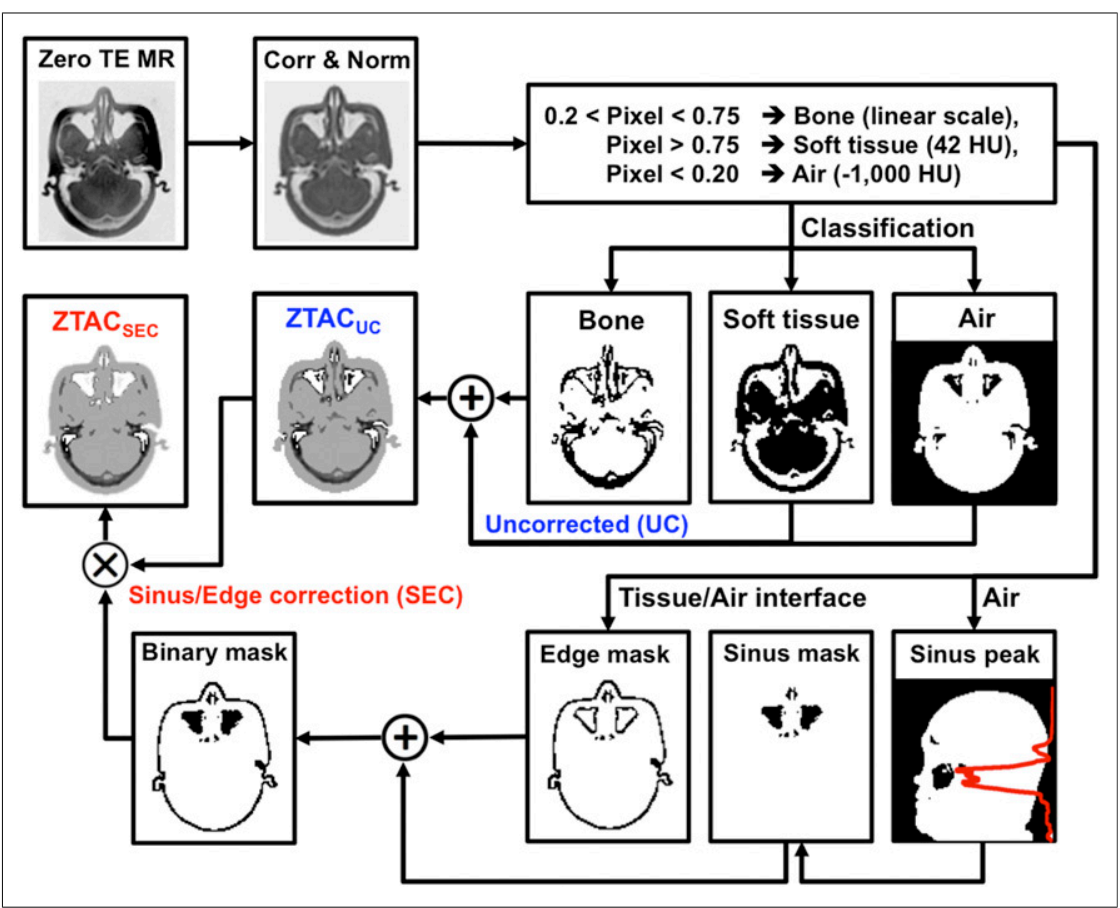

FIGURE 1. Workflow of generating pseudo-CT from ZTE images: ZTAC ${ }_{S E C}$ and ZTAC UC. In the binary mask, false-positive bone pixels were corrected to stay below $42 \mathrm{HU}$ (soft tissue).

\section{PET/CT}

PET/CT examinations were performed on a Discovery PET/CT (GE Healthcare) or Biograph HiRez 16 (Siemens Healthcare) scanner. The helical CT scans $(120 \mathrm{kVp}$; 105-599 mA; rotation time, $0.5 \mathrm{~s}$; pitch, 0.98 and 0.75 ; rotation, 39.37 and $34.45 \mathrm{~mm}$ for GE and Siemens systems, respectively) were obtained for PET AC. CT images were reconstructed with an axial FOV of 700 and $500 \mathrm{~mm}$, a slice thickness of 3.75 and $5.00 \mathrm{~mm}$, a matrix size of $512 \times 512$, and voxel sizes of $2.73 \times 2.73 \times$ 3.75 and $1.95 \times 1.95 \times 5.00 \mathrm{~mm}^{3}$ for GE and Siemens systems, respectively. For the head station, the FOV of $500 \mathrm{~mm}$ was enough to cover the whole head for all PET data acquired in PET/MRI.

\section{PET/MRI}

TOF PET/MRI acquisition was performed on a SIGNA PET/MR scanner (GE Healthcare). PET had a 600-mm transaxial FOV and 250-mm axial FOV, with a TOF timing resolution of approximately $400 \mathrm{ps}$ and average measured sensitivity of $22.65 \mathrm{cps} / \mathrm{kBq}$ (18). While PET data were acquired, 3-dimensional spoiled gradient echo T1-weighted images (repetition time, $\sim 4 \mathrm{~ms}$; first echo time/second echo time, 1.3/2.6 ms; flip angle, $5^{\circ}$; acquisition time, $18 \mathrm{~s}$ ) were acquired for PET AC, using the head and neck coil array. This sequence generated in-phase, out-ofphase, fat and water images using the Dixon method (transaxial FOV, $500 \mathrm{~mm}$; slice spacing, $2.6 \mathrm{~mm}$; matrix size, $256 \times 256 \times 120$; and voxel size, $\left.1.95 \times 1.95 \times 5.2 \mathrm{~mm}^{3}\right)$. Additionally, proton density-weighted ZTE images (transaxial FOV, 262 or $490 \mathrm{~mm}$; matrix size, $110 \times 110 \times$ 110 or $192 \times 192 \times 192$; voxel size, $2.4 \times 2.4 \times 2.4$ or $2.6 \times 2.6 \times$ $2.6 \mathrm{~mm}^{3}$ ) were acquired using the same head and neck coil array with 2 sequences (repetition time, $\sim 0.7 \mathrm{~ms}$; echo time, $0 \mathrm{~ms}$; flip angle, $0.6^{\circ}$; transmit/receive switching delay, $\sim 28 \mu \mathrm{s}$; readout duration, 440 or $384 \mu \mathrm{s}$; acquisition time, 41 or $84 \mathrm{~s}$ ). These were 3-dimensional radial acquisitions without slab selection, so there was no preferential scan direction. The FOV was isotropic as well as the spatial resolution. The center of $\mathrm{k}$-space was filled in by acquiring an additional set of low-frequency projections with lower gradient strengths (19). The smaller FOV ZTE image was acquired for dedicated brain imaging $(n=8)$, and the larger FOV ZTE image was acquired as a part of the whole-body imaging $(n=4)$. The contrast was equivalent between the 2 sequences: they used the same echo time, repetition time, and flip angles. The other key consideration when imaging bone with MRI is signal decay during the readout (20), and both sequences had nearly identical readout durations to yield nearly identical relative bone signal. These ZTE sequences were implemented and tested as potential commercial products.

\section{ZTAC $_{\text {Uc }}$}

Before sinus/edge correction was applied, ZTE images were converted to pseudo-CT as follows: first, ZTE values were bias-corrected (21) and normalized by the median tissue value. Then, ZTE values were converted to CT numbers (Hounsfield unit [HU]) by applying simple thresholds for air $(-1,000 \mathrm{HU}$ for ZTE $<0.2)$ and soft tissue $(42 \mathrm{HU}$ for ZTE $>0.75)$ and linear transformation for bone $(-2,000 \times$ [ZTE - 1] + $42 \mathrm{HU}$ for intermediate ZTE values) (Fig. 1). In $\mathrm{ZTAC}_{\mathrm{UC}}$-derived pseudo$\mathrm{CT}$, air/tissue interfaces (e.g., skin and trachea), air/tissue mixtures (e.g., sinus), and air/bone mixtures (e.g., mastoid) are typically misclassified. This was because these mixtures result in ZTE signal intensities that are in between air 


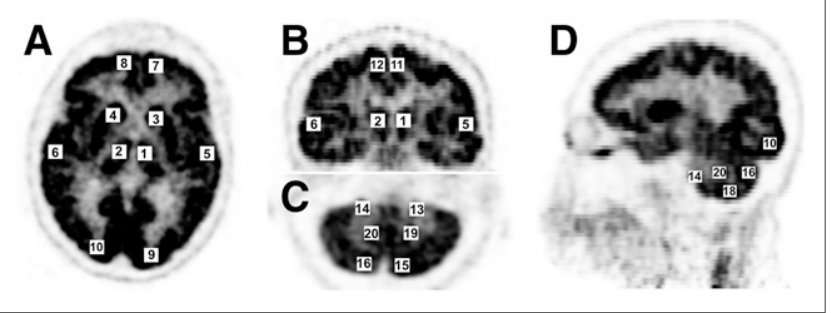

FIGURE 2. Localization of 20 VOls for VOI-based quantification: axial brain $(A)$, coronal brain $(B)$, axial cerebellum $(C)$, sagittal brain with sinus and cerebellum (D); left-right (1-2) thalamus, (3-4) corpus callosum, (5-6) temporal lobe, (7-8) frontal lobe, (9-10) occipital lobe, (11-12) cerebral cortex, (13-20) cerebellum at anterior (13-14), posterior (15-16), inferior (17-18), and center (19-20) positions.

and soft tissue, just like bone. For example, as shown in Figure 1, air/tissue mixtures in the sinus were partially misclassified as bone. This $\mathrm{ZTAC}_{\mathrm{UC}}$-derived pseudo-CT was a prior for $\mathrm{ZTAC}_{\mathrm{SEC}}$-derived pseudo-CT.

\section{ZTAC $_{\text {SEC }}$}

To reduce the effect of the misclassification above, sinus/edge correction was applied to $\mathrm{ZTAC}_{\mathrm{UC}}$-derived pseudo-CT where a binary mask including the sinus and air/tissue interfaces (edges) was estimated. The overestimated CT values in the binary mask were rescaled by the following formula to make them at least smaller than $42 \mathrm{HU}$ (soft tissue):

$$
\begin{aligned}
\mathrm{CT}(\mathrm{HU}) \text { in the binary mask }= & \min ([(1042 \times \mathrm{ZTE}) / 0.75 \\
& -1000], 42)
\end{aligned}
$$

The binary mask was generated as follows: first, the peak of the sinus was defined as the axial slice with the most internal air distribution, as measured on ZTE and shown by the red line in the sinus peak in Figure 1. Second, a square box $(3.5 \mathrm{~cm}$ above to $0.5 \mathrm{~cm}$ below the peak, covering a width of $\pm 2.5 \mathrm{~cm}$ ) was defined for the nasal area, in which the sinus was segmented and refined by morphologic processing (Fig. 1, sinus mask). Third, air/tissue edges were estimated and refined by edge detection and morphologic processing (Fig. 1, edge mask). Finally, the sinus mask and the edge mask were combined into a whole-brain binary mask. Note that $\mathrm{ZTAC}_{\mathrm{SEC}}$ was implemented and tested as a potential commercial product.

\section{ATAC}

The atlas-based pseudo-CT is the current implementation for brain PET AC in a SIGNA PET/MR scanner. Pseudo-CT using an atlas was prepared by segmenting the atlas (AT) into 4 tissue classes with air $(-1,000 \mathrm{HU}$ for outside of head), sinus $(-800 \mathrm{HU}$ for AT $<-200 \mathrm{HU})$, soft tissue (42 HU for intermediate AT), and bone (consistent HU for AT $>300 \mathrm{HU}$ with erosion). Before tissue classification, the single representative atlas was registered to patient MR (in-phase) images by contour-based rigid and nonrigid registration (8).

\section{CTAC}

Reference CT from PET/CT was prepared by removing its background (e.g., table) and registering to ZTE using Advanced Normalization Tools (22).

The 3 kinds of MRAC $\left(\right.$ ZTAC $_{\mathrm{UC}}, \mathrm{ZTAC}_{\mathrm{SEC}}$, and ATAC) pseudo-CTs and reference $\mathrm{CT}$ were converted to $\mathrm{AC}$ maps through bilinear transform (23), based on the CT energy of $120 \mathrm{kVp}$ for both pseudo-CT and CT. Attenuation templates of nonpatient components such as a table and head coil were developed by the vendor and added appropriately to the image space. The resulting PET volumes were named PET/ZTAC $\mathrm{UC}_{\mathrm{UC}}, \mathrm{PET} / \mathrm{ZTAC}_{\mathrm{SEC}}$,
PET/ATAC, and PET/CTAC, corresponding to the AC methods above, respectively.

\section{PET Reconstruction}

PET images were reconstructed using TOF ordered-subsets expectation maximization because it has demonstrated reduced errors caused by inaccurate MRAC (24) (point-spread function kernel derived from the PET detector response, 4 iterations, 28 subsets, a matrix size of $256 \times 256$ over $256 \mathrm{~mm}$ transaxial FOV, a voxel size of $1.0 \times 1.0 \times 2.78 \mathrm{~mm}^{3}$, with an in-plane gaussian filter of $3.0 \mathrm{~mm}$ in full width at half maximum followed by axial filtering with a 3-slice 1:4:1 kernel). The detector point-spread function, also known as the detector response function, was used to reduce detector blurring for resolution recovery (25). The filtering was a standard procedure recommended by the vendor for reducing high-frequency noise in PET images. The PET reconstruction with all implemented corrections (i.e., random, scatter, and normalization) was performed using an offline reconstruction toolbox (REL_1_26; GE Healthcare) in MATLAB (The MathWorks).

\section{Data Analysis}

Twenty volumes of interest (VOIs; $10 \times 10 \times 3$ voxels $=10 \times 10 \times$ $8.34 \mathrm{~mm}^{3} \approx 1 \mathrm{~mL}$ ) were placed close to cortical bone and higher uptake regions in PET images at the left and right side of thalamus, corpus callosum, temporal lobe, frontal lobe, occipital lobe, cerebral cortex, and cerebellum (Fig. 2). Specifically, 4 pairs of VOIs (anterior, posterior, center, and inferior) were localized in the cerebellum, considering its sensitivity to the accuracy of sinus segmentation (3). For each patient dataset, the VOIs were manually placed in similar positions throughout brain tissues, to preserve the original quality of patient-specific PET images for reliable patient-specific analysis. When PET/CTAC (PET reconstruction using CTAC) was used as a basis of comparison, PET uptake differences $(\mathrm{kBq} / \mathrm{mL})$ between PET/CTAC and PET/MRAC (PET uptake values with $\mathrm{ZTAC}_{\mathrm{UC}}, \mathrm{ZTAC}_{\mathrm{SEC}}$, or ATAC) were calculated for each VOI as follows:

$$
\begin{aligned}
\operatorname{Diff}(\mathrm{kBq} / \mathrm{mL})= & \text { mean }\left(\text { voxels of } \mathrm{VOI}_{\mathrm{MRAC}}\right) \\
& - \text { mean }\left(\text { voxels of } \mathrm{VOI}_{\mathrm{CTAC}}\right) \\
\operatorname{Diff}(\%)= & {\left[\text { mean }\left(\text { voxels of } \mathrm{VOI}_{\mathrm{MRAC}}\right)\right.} \\
& \left.- \text { mean }\left(\text { voxels of } \mathrm{VOI}_{\mathrm{CTAC}}\right)\right] \\
& / \text { mean }\left(\text { voxels of } \mathrm{VOI}_{\mathrm{CTAC}}\right) \times 100 .
\end{aligned}
$$

Patient-specific and VOI-specific results were presented across 12 patients and corresponding 20 VOIs through box plots that can show statistical distribution of PET uptake differences. A paired $t$ test was

TABLE 1

PET Uptake Differences Between Reference PET/CTAC and PET/MRAC (ZTAC ${ }_{U C}$, ZTAC $_{\mathrm{SEC}}$, and ATAC) Across All 240 VOls

\begin{tabular}{lrrr}
\hline & \multicolumn{2}{c}{ Difference } & \\
\cline { 2 - 3 } PET/MRAC & \multicolumn{1}{c}{$\mathrm{kBq} / \mathrm{mL}$} & $\%$ & \multicolumn{1}{c}{$P$} \\
\hline PET/ZTAC $_{U C}$ & $0.79 \pm 0.52$ & $5.6 \pm 3.5$ & $<0.01$ \\
PET/ZTAC $_{\mathrm{SEC}}$ & $0.03 \pm 0.33$ & $0.2 \pm 2.4$ & 0.23 \\
PET/ATAC $_{\text {PET }}$ & $-0.10 \pm 0.71$ & $-0.9 \pm 5.0$ & 0.03
\end{tabular}

Differences are mean $\pm \mathrm{SD}$. A paired $t$ test was performed for pair of PET/CTAC and PET/MRAC. 


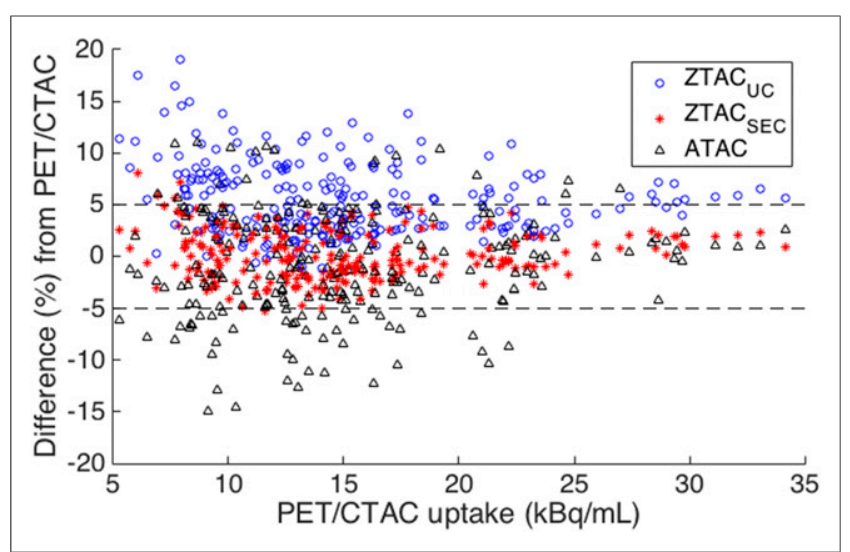

FIGURE 3. Bland-Altman plot of 240 VOls of 12 patients for PET uptake differences (\%) with MRAC (ZTAC ${ }_{U C}, Z_{\text {ZTAC }}$ SEC ATAC) from PET

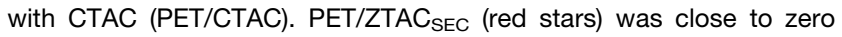
mean difference mostly within $\pm 5 \%$ boundaries.

performed for comparing the PET uptake differences of PET/MRAC from reference PET/CTAC. A $P$ value of less than 0.05 was considered to indicate statistical significance.

A histogram was used to show the distribution of voxel-based PET uptake differences (PET/CTAC - PET/MRAC) within the SUV (imagederived uptake $[\mathrm{MBq} / \mathrm{mL}] /$ injection dose $[\mathrm{MBq}] \times$ patient's weight $[\mathrm{g}])$ range of $0.5-15.0(\mathrm{~g} / \mathrm{mL})$ across all subjects. Additionally, pseudo-CT, AC maps, and PET images of representative patients were illustrated according to the AC methods, specifically focusing on the cerebellum and mastoid.

\section{RESULTS}

The mean uptake difference $( \pm \mathrm{SD})$ of PET/ZTAC $\mathrm{SEC}_{\mathrm{S}}$ from PET/CTAC was $0.2 \% \pm 2.4 \%(P=0.23)$, the difference of $\mathrm{PET}_{\text {ZTAC }}$ UC was $5.6 \% \pm 3.5 \%(P<0.01)$, and the difference of PET/ATAC was $-0.9 \% \pm 5.0 \%(P=0.03)$ (Table 1). All $t$ tests were statistically significant except the $t$ test between $\mathrm{PET}_{\text {ZTAC }}$ SEC and PET/CTAC, implying PET/ZTAC SEC $_{\text {was }}$ statistically similar with PET/CTAC. In the Bland-Altman plot (Fig. 3), most of the circles with $\mathrm{ZTAC}_{\mathrm{UC}}$ were positioned in positive areas, indicating substantial overestimation of PET uptake in comparison with CTAC; whereas most of the triangles with ATAC were more scattered over negative areas (SD, 5.0\%) than the circles with $\mathrm{ZTAC}_{\mathrm{UC}}(\mathrm{SD}, 3.5 \%)$. On the contrary, the stars with $\mathrm{ZTAC}_{\mathrm{SEC}}$ were positioned within the range of $\pm 5 \%$ with the smallest variation (SD, 2.5\%).

However, the results were patient- and VOI-location dependent. In Figure 4A, the improved inter- and intrapatient variations by $\mathrm{ZTAC}_{\mathrm{SEC}}$ were consistent with the overall result as above. In terms of interpatient variation, PET uptake differences with $\mathrm{ZTAC}_{\mathrm{SEC}}$ in the boxes (i.e., 25th-75th percentiles) of all patients stayed within the boundaries of $\pm 5 \%$. Also, in terms of intrapatient variation, the 25 th-75th percentiles with ZTAC $_{\text {SEC }}$ usually had a smaller or comparable range compared with those with $\mathrm{ZTAC}_{\mathrm{UC}}$ and ATAC. In Figure 4B, the reduction of VOIdependent variations by $\mathrm{ZTAC}_{\mathrm{SEC}}$ was also consistent with the overall result as above: PET uptake differences with $\mathrm{ZTAC}_{\mathrm{SEC}}$ in the boxes of all VOIs stayed within the boundaries of $\pm 5 \%$. Specifically, ZTAC $_{\text {SEC }}$ improved PET quantification accuracy $(0.6 \% \pm 2.7 \%$; range, $-6.6 \%-8.1 \% ; P<0.01)$ significantly in the cerebellum at the level of sinus, compared with the accuracy of $\mathrm{ZTAC}_{\mathrm{UC}}(8.1 \% \pm 3.5 \%$; range, $1.3 \%-19.0 \% ; P<0.01)$ and that of ATAC $(-4.1 \% \pm 4.3 \%$; range, $-15.0 \%-4.6 \% ; P<0.01)$.

The histogram of voxel-based PET uptake differences (PET/ MRAC - PET/CTAC) across all patients demonstrated that ZTAC $_{\mathrm{SEC}}$ reduced the magnitude and variation of errors substantially, compared with those of $\mathrm{ZTAC}_{\mathrm{UC}}$ and ATAC (Fig. 5). The 25 and 75 percentiles (vertical yellow lines) demonstrated that the uptake differences of PET/ZTAC ${ }_{\mathrm{SEC}}$ and PET/ATAC were symmetrically distributed from the origin (zero error), though those of PET/ZTAC $\mathrm{UC}_{\mathrm{UC}}$ were mostly distributed in the positive (overestimated) error zone.

The voxel-based comparison was consistent with the patientdependent comparison (Fig. 6). In Figure 6A (patient 5, sagittal view), $\mathrm{ZTAC}_{\mathrm{SEC}}$-derived pseudo-CT (first row) was more consistent with reference $\mathrm{CT}$ at the sinus region (arrows), whereas ZTAC $_{\mathrm{UC}^{-}}$-derived pseudo-CT illustrates misclassified bone signals and ATAC-derived pseudo-CT illustrates geometric offsets from reference $\mathrm{CT}$ at anatomic structures such as sinus and skull. Consistently, $\mathrm{ZTAC}_{\mathrm{SEC}}$-derived $\mathrm{AC}$ maps (second row) illustrate the improved sinus, compared with $\mathrm{ZTAC}_{\mathrm{UC}^{-}}$and ATAC-derived AC maps. As a result, ZTAC $_{\text {SEC }}$ improved PET quantification in the cerebellum (third row, arrows) at the level of sinus without substantial over- or underestimation whereas PET/ZTAC $_{U C}$ was consistently overestimated throughout the brain but more substantially at the level of sinus and PET/ATAC was overestimated at skull top but underestimated at the skull base. However, in Figure 6B (patient 5, coronal view), misclassified bone signals at the mastoid were not fixed in $\mathrm{ZTAC}_{\mathrm{SEC}}$-derived
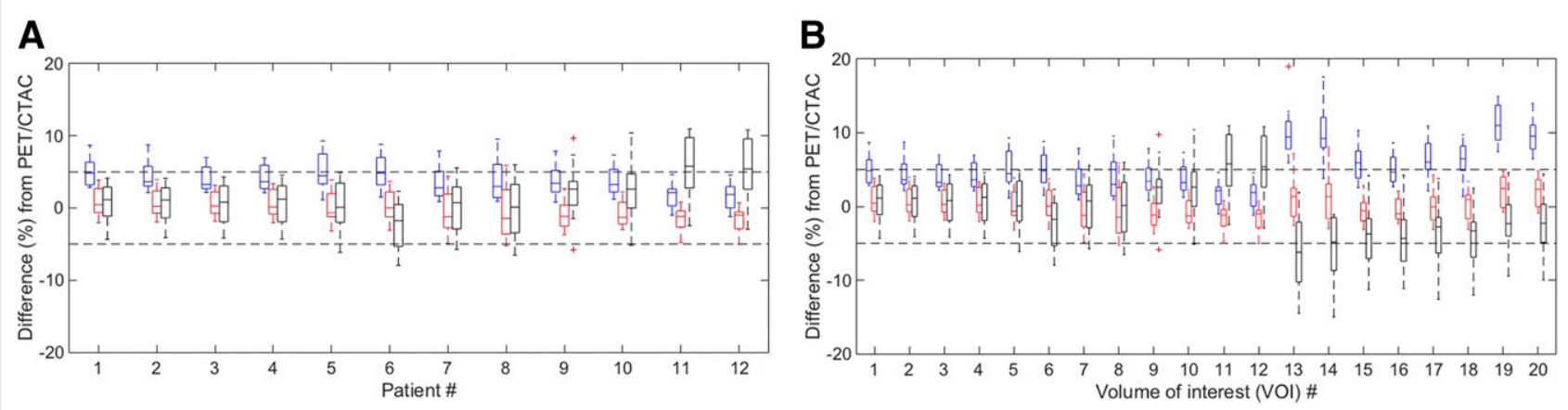

FIGURE 4. Box plots of PET uptake differences (\%) for 12 patients across 20 VOIs (A) and for 20 VOls across 12 patients (B) between PET/CTAC and PET/MRAC (left, blue: ZTAC $U$; ; middle, red: ZTAC $_{S E C}$; right, black: ATAC). Tops and bottoms of each box are 25th and 75th percentiles of samples, respectively, with median and outlier (+ sign, $>1.5 \times$ interquartile range). 

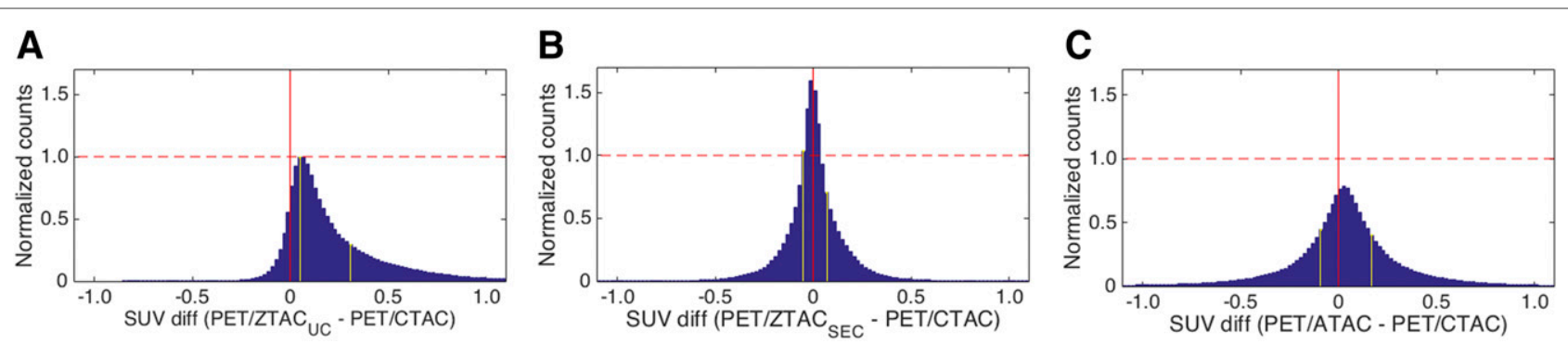

FIGURE 5. Histograms of voxel-based PET uptake differences (PET/MRAC - PET/CTAC) across all patients using voxels within SUV range of

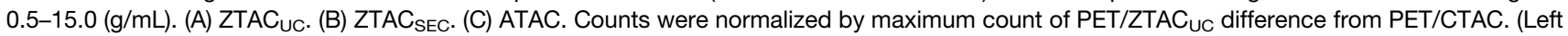
to right) Yellow lines indicate 25th and 75th percentiles.

pseudo-CT (first row) because the binary mask did not include the mastoid region for correction (Fig. 1). The impact of the mastoid misclassification in ZTAC $_{\text {SEC }}$ was illustrated as slightly overestimated PET uptake near the mastoid, whereas the impact of the mastoid misclassification was negligible in the cerebellum. In Figures 6C and 6D (patient 8), the similar observations were visually accounted for and specifically in the cerebellum at the level of sinus.
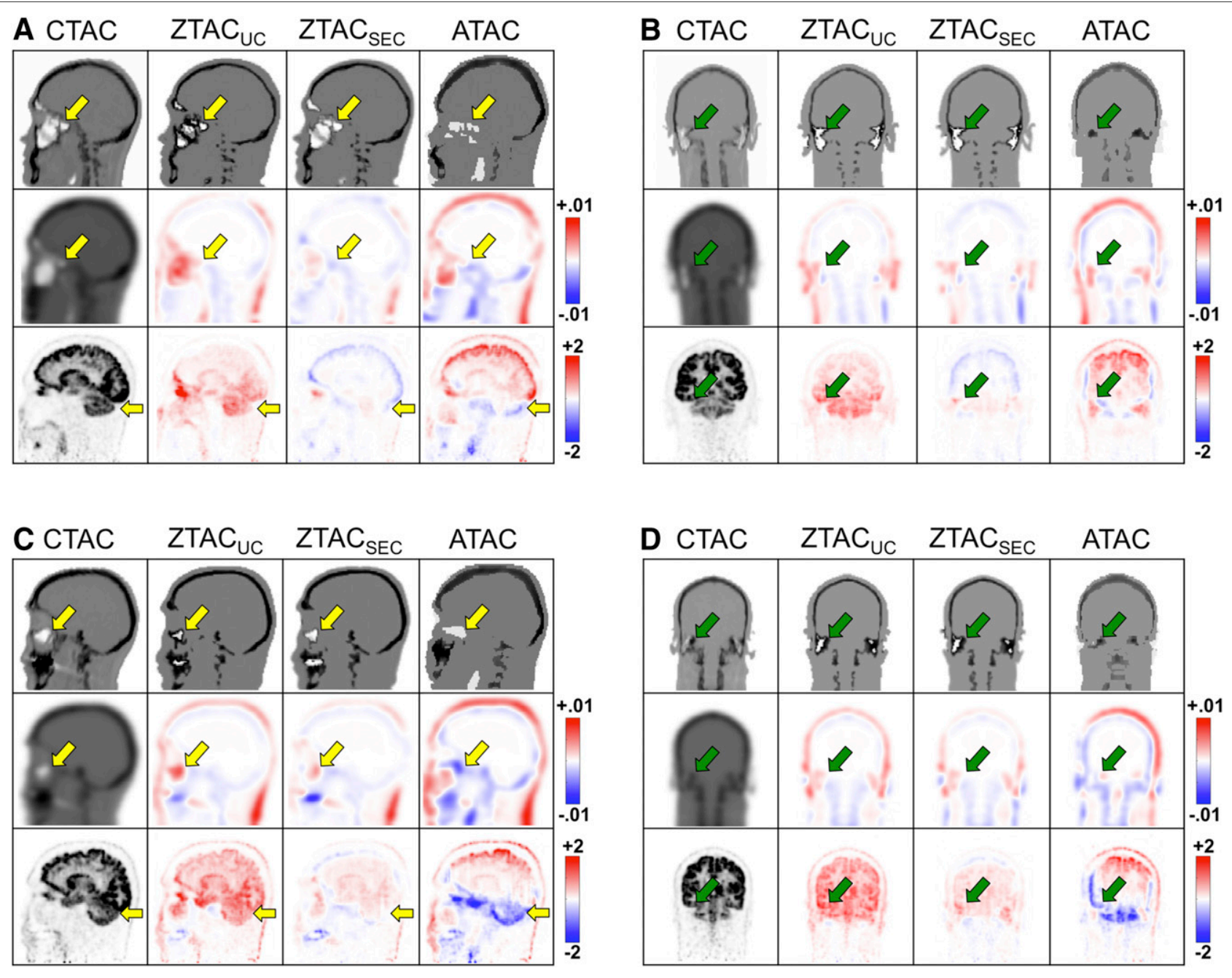

FIGURE 6. Patients 5 ( $A$ and $B$ ) and $8(C$ and $D)$ in coronal and sagittal views for $C T$ and pseudo-CTs (first row), attenuation-correction ( $A C$ ) maps (second row), and corresponding PET images (third row) according to AC methods. Second and third rows illustrate difference images on second through fourth columns for pseudo-CT-derived AC maps from CT-derived AC maps (unit, $\mathrm{cm}^{-1}$ ) and PET/MRAC from PET/CTAC (unit, SUV), respectively. Arrows indicate sinus, mastoid, and cerebellum. 


\section{DISCUSSION}

The ZTAC $_{\text {SEC }}$ was implemented for a potential commercial translation, overcoming the major challenge of ZTAC by correcting misclassified bone signals due to air/tissue/bone mixtures or interfaces. Our results demonstrated that $\mathrm{ZTAC}_{\mathrm{SEC}}$ (mean uptake difference, $0.2 \% \pm 2.4 \%$ ) outperformed $\mathrm{ZTAC}_{\mathrm{UC}}$ $(5.6 \% \pm 3.5 \%)$ and ATAC $(-0.9 \% \pm 5.0 \%)$ for PET quantification accuracy and precision, compared with CTAC. Specifically, a significant improvement with $\mathrm{ZTAC}_{\mathrm{SEC}}$ was found in the cerebellum at the level of sinus, the most vulnerable area affected by inaccurate MRAC $(3,16)$.

Interpatient anatomic and bone-density variability played a key role in the performance of each method (24). The ATAC method relied on a single-atlas CT, which could not capture the range of interpatient variability in our subjects. This caused the error variation of PET/ATAC (SD, 5.0\%) to be larger than those of PET/ZTAC $\mathrm{SEC}_{\mathrm{SE}}(\mathrm{SD}, 2.4 \%)$ and PET/ZTAC $\mathrm{UC}_{\mathrm{UC}}(\mathrm{SD}$, $3.5 \%)$. ZTAC reduced the variation by removing the need of registration and by estimating bone density through a linear conversion of ZTE bone to $\mathrm{CT}$ values. The remaining patientspecific error variation of PET/ZTAC $\mathrm{SEC}_{\mathrm{SE}}(\mathrm{SD}, 2.4 \%)$ was large because sinus/edge correction could not consistently capture patient-specific mixtures of air/tissue/bone in the sinuses. In terms of the interregional variability, the variability was the most severe in the cerebellum at the level of sinuses for both PET/ATAC and PET/ZTAC ${ }_{\mathrm{UC}}$. Because air, soft tissue, and bone structures are mixed in the sinuses, it was a challenge to achieve accurate registration for ATAC (Fig. 6) and to classify the mixed signals accurately for $\mathrm{ZTAC}_{\mathrm{UC}}$.

In a prior study using a preliminary ZTAC method (16), similar to the $\mathrm{ZTAC}_{\mathrm{UC}}$, the overall error $(-0.1 \% \pm 2.3 \%)$ was smaller than the error of $\mathrm{ZTAC}_{\mathrm{UC}}(5.6 \% \pm 3.5 \%)$. The larger error of $Z_{\mathrm{ZTAC}} \mathrm{UC}_{\mathrm{C}}$ could be derived from the following differences because our ZTE-CT conversion was optimized for $\mathrm{ZTAC}_{\mathrm{SEC}^{-}}$ derived pseudo-CT: the segmentation thresholds for bone class (our ZTACs vs. preliminary ZTAC: $0.2<$ ZTE $<0.75$ vs. $0.25<$ ZTE $<0.85)$ and the slope of linear correlation between ZTE and CT values (our ZTACs vs. preliminary ZTAC: 2,000 vs. $2,400)$. The overall error of $\mathrm{ZTAC}_{\mathrm{SEC}}$ in our study $(0.2 \% \pm$ $2.4 \%$ error) was comparable to that of the preliminary ZTAC study. However, for the cerebellum, which was the most vulnerable to AC errors in the sinus, the average error of $\mathrm{ZTAC}_{\mathrm{SEC}}$ $(0.6 \% \pm 2.7 \%)$ was much lower than that of the preliminary ZTAC study $(3.31 \% \pm 1.70 \%)(23)$.

There were several limitations of our study: a limitation was the small number of patients $(n=12)$. Also, the PET data acquisition was not optimized for dedicated brain PET scans that are typically obtained with a longer acquisition time. However, we used brain PET reconstruction with a small FOV reconstruction (256 $\mathrm{mm}$ in the transaxial view) and a high number of iterations, resulting in a smaller voxel size $(1.0 \times 1.0 \times$ $2.78 \mathrm{~mm}^{3}$ ) for brain PET quality assurance. Although both the small voxel size and the shorter scan time might cause a low signal-to-noise ratio, this problem was mitigated in the VOIbased analysis by averaging the values of $10 \times 10 \times 3$ (300) voxels located in higher activity regions. Also, 2 different ZTE MRI protocols were used, but the differences between these protocols are likely negligible for ZTAC processing: they were designed to provide nearly identical bone-to-soft-tissue contrast, the key parameter in the pseudo-CT generation method, and provided similar voxel sizes. We expect the impact of resolution differences on MRAC accuracy to be small, considering that AC maps were substantially blurred due to gaussian postfiltering with $10 \mathrm{~mm}$ in full width at half maximum (this AC map filtering was a standard procedure recommended by the vendor). We observed no consistent differences in the AC maps between the protocols. Another limitation was that 2 different CT scanners might cause an impact on the quality of reference $\mathrm{CT}$ in the course of registration. Considering the low resolution of $\mathrm{AC}$ maps $\left(4.69 \times 4.69 \mathrm{~mm}^{2}\right)$, however, the potential impact of CT quality variation was likely suppressed through gaussian postfiltering with $10 \mathrm{~mm}$ in full width at half maximum at the final step of AC map generation. Additionally, the manual localization of VOIs might cause operator-dependent results. To mitigate potential errors, a single operator performed all localization. Compared with using a brain template, using VOIs with small sizes $(\approx 1 \mathrm{~mL})$ and higher uptake preserved the sensitivity of localized errors with regards to the bone and sinus, and reduced potential bias of errors amplified by relatively lower uptake regions (24). Finally, the current correction algorithm did not estimate the mastoid with air/bone mixtures, as illustrated in Figure 1, resulting in slight overestimation of PET uptake near the mastoid. If mastoid regions could be estimated accurately, the accuracy of $\mathrm{ZTAC}_{\mathrm{SEC}}$ would be more improved near the mastoid region.

\section{CONCLUSION}

We evaluated the newly implemented ZTAC $_{\text {SEC }}$ for a potential commercial product. Our results demonstrated that ZTAC $_{\text {SEC }}$ improved PET quantification accuracy over $\mathrm{ZTAC}_{\mathrm{UC}}$ and ATAC, particularly in the cerebellum at the level of sinus.

\section{DISCLOSURE}

This project was supported in part by grant from GE Healthcare. No other potential conflict of interest relevant to this article was reported.

\section{ACKNOWLEDGMENTS}

We thank Michel Tohme, PhD, Mehdi Khalighi, PhD, and Floris Jansen, $\mathrm{PhD}$ of GE Healthcare for the support of the offline PET/MR reconstruction toolkit. We also thank Vahid Ravanfar, BS, for his support in obtaining the PET/MRI scans.

\section{REFERENCES}

1. Iagaru A, Mittra E, Minamimoto R, et al. Simultaneous whole-body time-offlight ${ }^{18}$ F-FDG PET/MRI: a pilot study comparing SUVmax with PET/CT and assessment of MR image quality. Clin Nucl Med. 2015;40:1-8.

2. Nensa F, Beiderwellen K, Heusch P, Wetter A. Clinical applications of PET/ MRI: current status and future perspectives. Diagn Interv Radiol. 2014;20:438447.

3. Visvikis D, Monnier F, Bert J, Hatt M, Fayad H. PET/MR attenuation correction: where have we come from and where are we going? Eur J Nucl Med Mol Imaging. 2014;41:1172-1175.

4. Bezrukov I, Mantlik F, Schmidt H, Scholkopf B, Pichler BJ. MR-based PET attenuation correction for PET/MR imaging. Semin Nucl Med. 2013;43:45-59.

5. Martinez-Möller A, Souvatzoglou M, Delso G, et al. Tissue classification as a potential approach for attenuation correction in whole-body PET/MRI: evaluation with PET/CT data. J Nucl Med. 2009;50:520-526.

6. Du J, Carl M, Bydder M, Takahashi A, Chung CB, Bydder GM. Qualitative and quantitative ultrashort echo time (UTE) imaging of cortical bone. J Magn Reson. 2010;207:304-311. 
7. Andersen FL, Ladefoged CN, Beyer T, et al. Combined PET/MR imaging in neurology: MR-based attenuation correction implies a strong spatial bias when ignoring bone. Neuroimage. 2014;84:206-216.

8. Wollenweber SD, Ambwani S, Delso G, et al. Evaluation of an atlas-based PET head attenuation correction using PET/CT \& MR patient data. IEEE Trans Nucl Sci. 2013;60:3383-3390.

9. Burgos N, Cardoso MJ, Thielemans K, et al. Attenuation correction synthesis for hybrid PET-MR scanners: application to brain studies. IEEE Trans Med Imaging. 2014;33:2332-2341.

10. Koesters T, Friedman KP, Fenchel M, et al. Dixon sequence with superimposed model-based bone compartment provides highly accurate PET/MR attenuation correction of the brain. J Nucl Med. 2016;57:918-924.

11. Huynh T, Gao Y, Kang J, et al. Estimating CT image from MRI data using structured random forest and auto-context model. IEEE Trans Med Imaging. 2016;35:174-183.

12. Berker Y, Li Y. Attenuation correction in emission tomography using the emission data: a review. Med Phys. 2016;43:807-832.

13. Wiesinger F, Sacolick LI, Menini A, et al. Zero TE MR bone imaging in the head. Magn Reson Med. 2016;75:107-114.

14. Larson PEZ, Han MS, Krug R, et al. Ultrashort echo time and zero echo time MRI at 7T. MAGMA. 2016;29:359-370.

15. Ladefoged CN, Benoit D, Law I, et al. Region specific optimization of continuous linear attenuation coefficients based on UTE (RESOLUTE): application to PET/MR brain imaging. Phys Med Biol. 2015;60:8047-8065.
16. Sekine T, Ter Voert EE, Warnock G, et al. Clinical evaluation of ZTE attenuation correction for brain FDG-PET/MR imaging: comparison with atlas attenuation correction. J Nucl Med. 2016;57:1927-1932.

17. Su Y, Rubin BB, McConathy J, et al. Impact of MR-based attenuation correction on neurologic PET studies. J Nucl Med. 2016;57:913-917.

18. Grant AM, Deller TW, Khalighi MM, Maramraju SH, Delso G, Levin CS. NEMA NU 2-2012 performance studies for the SiPM-based ToF-PET component of the GE SIGNA PET/MR system [abstract]. Med Phys. 2016;43:2334.

19. Wu Y, Dai G, Ackerman JL, et al. Water- and fat-suppressed proton projection MRI (WASPI) of rat femur bone. Magn Reson Med. 2007;57:554-567.

20. Rahmer J, Bornert P, Groen J, Bos C. Three-dimensional radial ultrashort echotime imaging with T2 adapted sampling. Magn Reson Med. 2006;55:1075-1082.

21. Tustison NJ, Avants BB, Cook PA, et al. N4ITK: improved N3 bias correction. IEEE Trans Med Imaging. 2010;29:1310-1320.

22. Avants BB, Tustison N, Song G. Advanced normalization tools (ANTS). Insight J. 2009;2:1-35.

23. Lonn AHR. Evaluation of method to minimize the effect of X-ray contrast in PET-CT attenuation correction. 2003. IEEE Nucl Sci Symp Conf Rec. 2003;3:2220-2221.

24. Yang J, Jian Y, Jenkins N, et al. Quantitative evaluation of atlas-based attenuation correction for brain PET imaging in an integrated time-of-flight PET/MRI system. Radiology. 2017;284:169-179.

25. Rahmim A, Qi J, Sossi V. Resolution modeling in PET imaging: theory, practice, benefits, and pitfalls. Med Phys. 2013;40:064301. 